\title{
Treatment of Acute Anxiety Episodes in Patients Using a Fast-Acting Beta Blocker - Scopolamine Combination Drug
}

\author{
Thomas $\mathrm{T}^{1}$, Benjamin $\mathrm{AB}^{2}$ and Dooley $\mathbf{T P}^{3 *}$ \\ ${ }^{1}$ National Centers for Pain Management and Research, \\ USA \\ ${ }^{2}$ Sterling Care Psychiatric Group in Ventura County, USA \\ ${ }^{3}$ Trends in Pharma Development LLC, USA \\ *Corresponding author: Dooley TP, Trends in \\ Pharma Development LLC, 7100 Cabin Lane, Pinson, AL \\ 35126, USA
}

Received: September 11, 2017; Accepted: November 09, 2017; Published: November 16, 2017

\begin{abstract}
A new class of anti-anxiety medications has been developed and patented that are dual drug combinations of a beta blocker and an antimuscarinic agent. The PanX® drug combinations were designed as pro re nata (prn) alternatives to addictive benzodiazepines, without the use of addictive Controlled Substances. The beta blocker (beta adrenergic receptor antagonist) addresses the sympathetic cardiovascular symptoms and the antimuscarinic agent (muscarinic receptor antagonist) addresses the parasympathetic CNS and other non-cardiovascular symptoms. Here we present a case series of three adult patients, who were being assessed and/or treated in a pain management clinic, and who reported that they experienced acute anxiety episodes. Pain management is often confounded by comorbid anxiety disorders, for which addictive benzodiazepines are the standard-of-care for prn treatments. The patients consented to a physician-sponsored study using a prescription compounded medication. They self-administered an atenolol - scopolamine $\mathrm{HBr}$ drug combination either during or immediately prior to the anticipated acute anxiety symptoms. The patients completed a questionnaire thereafter of their symptoms and the effects and side effects of the medicine. The dual drug combination was well tolerated and resulted in a self-perceived calming effect within 6 - 60 minutes that persisted for approximately $2-6$ hours in the three patients. Furthermore, all three patients reported that they would be willing to use this new (compounded) medication for anxiety treatments beyond the physician-sponsored study.
\end{abstract} PanX

Keywords: Atenolol; Scopolamine; Anxiety; Panic; Pain management;

\section{Introduction}

Anxiety disorders affect approximately 40 million people in the USA alone. Many of them experience episodes of acute anxiety, for which they often use benzodiazepines or alcohol for relief. Benzodiazepines, such as Xanax (alprazolam) or Klonopin (clonazepam), are the FDA-approved and effective standard-of-care medicines for acute anxiety. But, they pose many safety problems for patients and physicians. These include addiction, the potential for abuse, risk of death in combination with opioids, tolerance, and impaired cognition and reaction time [1-4]. The only other FDAapproved products for the short-term treatment of anxiety include Buspar (buspirone) or the antihistamine Vistaril (hydroxyzine). However, neither drug is generally considered by psychiatrists to be substantially effective at treating acute anxiety episodes. Overall, physicians have a very short list of FDA-approved options for the prn treatment of acute anxiety, and each of those current options is less than ideal.

In chronic and acute pain management there are often confounding mental health issues to consider. How does a physician ethically treat a patient manifesting both pain and coincident anxiety symptoms, while attempting to minimize the risk for addiction and/ or adverse drug-drug interactions? One would strongly prefer non- addicting active ingredients to treat both conditions in the same patient. The FDA issued a warning notice in 2016 concerning the coincident use of benzodiazepines and opioids that can result in an increased risk of death by overdose. Opioids are the frontline tools in treating moderate-to-severe pain [5]. And, there are relatively few effective pain medications that are not opioids that can be used for some moderate-to-severe conditions (e.g., gabapentin, pregabalin, and ketamine), as well as NSAIDs and acetaminophen for mildto-moderate pain. The opioid class of medicines is also prone to abuse and addiction, like the benzodiazepine class. Furthermore, opioid abusers and addicts undergo severe withdrawal symptoms, including acute anxiety and seizures, which are typically treated prn with benzodiazepines. Thus, pain management, mental health, and primary care physicians are in great need of new solutions to this benzodiazepine-opioid dilemma for the management of pain plus anxiety [6]. One new solution is introduced in this study.

Here we present a case series of three patients assessed and/ or treated in a pain management clinic who used a new approach, a PanX drug combination of a beta blocker and an antimuscarinic (motion sickness) agent. These medicines are a patented new class of anxiolytics intended especially for the prn treatment of the symptoms of acute anxiety [7]. Beta blockers inhibit the binding 
of catecholamine's (e.g., epinephrine) to beta adrenergic receptors. Propranolol or atenolol, among others, have been prescribed offlabel for decades to suppress the cardiovascular symptoms of acute anxiety, for instance tachycardia and palpitations in performance anxiety. However, a beta blocker alone does not address the CNS and other non-cardiovascular symptoms. Therefore, a muscarinic receptor antagonist, of which scopolamine is one of the most potent, is included in the new drug combinations. Scopolamine affects the CNS symptoms of anxiousness, fear, and avoidance, plus nausea and vomiting that are components of motion sickness. The drug combos were designed to address the sympathetic (beta blocker) and parasympathetic (antimuscarinic) symptoms of acute anxiety episodes, yet without using any addictive Controlled Substances. Both classes of active ingredients within the new drug combinations are known to be historically safe and non-addicting.

\section{Methods}

Three adult patients were assessed in a pain management clinic and reported acute anxiety symptoms. Each patient was informed of a commercially-available compounded medicine intended for the treatment of anxiety symptoms. Then, each provided written consent to participate in a physician-sponsored open label study related to the treatment of his/her anxiety symptoms and for the use of his/ her results in research and/or publications. Each was prescribed the compounded PanX drug combination of atenolol $25 \mathrm{mg}$ scopolamine $\mathrm{HBr} 0.2 \mathrm{mg}$ in an orally-disintegrating triturate tablet (in mannitol) that was prepared by National Pain Custom Pharmacy (AL). A patient questionnaire developed by our psychiatrist coauthor was provided to record his/her symptoms, and the effects and side effects of the drug treatment. The patients and medical staff were not compensated for participating.

\section{Case Presentation}

Patient $\mathrm{A}$ is a 35 year old Caucasian male who was being assessed in a pain management clinic for chest pain, perhaps related to cough or palpitations, and panic attacks. He stated his acute anxiety symptoms included nervousness, panic, palpitations, feelings of shortness of breath and choking, and the fear of dying. The symptoms typically last for about 30 minutes. He reported that he commonly uses a beta blocker. He took the drug combo mucosal by sublingual delivery and immediately prior to when he expected the anxiety symptoms to develop. He described a calming effect with full remission of his anxiety symptoms, and without side effects of dry mouth, fatigue, blurred vision, or confusion. However, he did report some dizziness and sleepiness. The patient recorded that he first perceived a beneficial effect within 6 minutes, and the effects persisted for 6 hours. Patient A expressed interest in using this medicine again after the physiciansponsored study.

Patient B is a 56 year old African-American female who was being treated in a pain management clinic for peripheral neuropathic pain, but also mentioned experiencing acute anxiety episodes. She stated her acute anxiety symptoms included intense anxiety (anxiousness), nervousness, sweating, headache, nausea, vomiting, and feelings of shortness of breath and numbness/tingling. The symptoms typically last for about 60 minutes. The patient's current use of the opioids tramadol and buprenorphine (in Suboxone) were verified by analytical chemistry using LC/MS. Furthermore, the patient reported a history of tizanidine, Soma (carisoprodol), and Seroquel (quetiapine). On three occasions on separate days she self-administered the drug combo orally and immediately prior to when she expected the symptoms to develop. On all three occasions she described a calming effect with some remission of her anxiety symptoms, and without side effects of dizziness, fatigue, sleepiness, blurred vision, or confusion. However, she did report dry mouth as a side effect. The patient recorded that she first perceived a beneficial calming effect within 60 minutes, and the effects persisted for 4 hours. Patient B expressed interest in using this medicine again after the physician-sponsored study.

Patient $\mathrm{C}$ is a 40 year old Caucasian female who was being assessed in a pain management clinic for acute anxiety episodes (situational anxiety). She stated her acute anxiety symptoms included intense anxiety (anxiousness), nervousness, and palpitations. The symptoms typically last for about 60-90 minutes. She reported that she was not taking any anxiolytic medicine at the time. She self-administered the drug combo mucosal by oral disintegration and after she started experiencing the symptoms. She described a calming effect with full resolution of palpitations and partial reduction in anxiety, and without side effects of dry mouth, dizziness, fatigue, sleepiness, blurred vision, or confusion. However, she did report some numbness or tingling of the skin of her forearms. The patient recorded that she first perceived a beneficial effect within 10-15 minutes, and the effects persisted for 2 hours. Patient $\mathrm{C}$ described the calming effect as "feeling slow in a pleasant way" and that "time seemed to go faster". Patient C expressed interest in using this medicine again after the physiciansponsored study.

\section{Discussion}

The PanX drugs were developed and patented to provide prn alternatives to addictive benzodiazepines, without the use of any addictive active ingredients or Controlled Substances [7]. Atenolol is a beta- 1 selective antagonist of adrenalin that has been shown to be beneficial in performance anxiety, alcohol withdrawal, and flight phobia [8-12]. Scopolamine has been known for over a century to produce a calming effect in psychiatric patients [13]. Scopolamine is a high potency muscarinic receptor antagonist that likely affects anxiety symptoms via the M2 and/or M1 receptors of the CNS [14].

Beta blockers and antimuscarinic agents were combined not only for pharmacologic targeting of the beta-1 and M2/M1 receptors, but also in view of historic safety. Both classes of drugs have been used in millions of patients over five decades and are known to be safe. Neither class is addictive or prone to abuse. In addition, the high prevalence of use strongly suggests that both classes of medicines have been routinely and concurrently used in the same patient(s) as separate prescriptions and/or OTC drugs. For instance, a patient on a beta blocker for hypertension, might also takes an antimuscarinic for motion sickness or nausea. Yet, there is no literature or post-market evidence in the population of producing side effects that extend beyond those inherent within either of the monotherapies.

This case series documents for the first time an open label study of a new compounded combination drug regarding anxiolytic efficacy and side effects in multiple adult patients assessed and/or treated in a pain management clinic. The drug combo produced a fast-acting 
and beneficial calming affect for multiple hours by both oral and mucosal routes of administration. Plus, it demonstrated benefit when administered either during anxiety episodes or immediately prior to anticipate symptoms. It is possible that the oral dosing in patient B, relative to the other patients who used oral mucosal delivery, resulted in a slower perception of onset of a beneficial effect (i.e., 60 minutes oral vs. 6-15 minutes mucosal). Oral delivery is known in general in pharmacology to result in a "lower and slower" effect than mucosal delivery, due to GI absorption and first-pass hepatic metabolism.

The medicines were well tolerated in all three patients. None of the patients described any serious adverse effects, although each noted minor side effects that were unique to the individual. There was no evidence of impairment of cognition. All three indicated that they were interested in using the drug combo after the physiciansponsored study.

There are three FDA-approved approaches for short-term treatment of acute anxiety: benzodiazepines, buspirone, and hydroxyzine. Benzodiazepines are effective, but they have many detrimental side effects. And, there is a general perception by many healthcare providers and patients that buspirone and hydroxyzine aren't effective. Therefore, the patented drug combinations are a promising new class of prn anxiolytics. This non-benzodiazepine alternative approach is appealing for the symptomatic treatment of anxiety disorders (e.g., social anxiety disorder, panic disorder, PTSD). This is especially true in the management of chronic and acute pain patients on opioids, because the FDA has issued a warning in 2016 concerning the risk of death due to coincident use of benzodiazepines and opioids. A beta blocker - antimuscarinic combination drug can overcome this serious concern.

\section{Acknowledgement and Disclosure}

We are grateful for the assistance of Daniel Moylan (study coordinator) and Dr. Alex Pisaturo (physician). Compounded medications are not US FDA-approved, but are subject to Section 503A of the FD\&C Act of 2013. The PanX drug combos are exclusive to TPD LLC (www.PanX.us) and are protected by three US patents and international patent applications. Dr. Thomas is a pain management specialist with National Centers for Pain Management \& Research (\& Alabama Pain Physicians \& National Pain Custom Pharmacy). Dr. Benjamin is a staff psychiatrist of Sterling Care Psychiatric Group and a consultant to TPD LLC. Dr. Dooley is an employee and shareholder of TPD LLC. This research did not receive any specific grant from funding agencies in the public, commercial, or not-for-profit sectors.

\section{References}

1. Greenblatt DJ, Shader RI. Dependence, tolerance, and addiction to benzodiazepines: clinical and pharmacokinetic considerations. Drug Metab Rev. 1978; 8: 13-28.

2. Griffiths RR, Johnson MW. Relative abuse liability of hypnotic drugs: conceptual framework and algorithm for differentiating among compounds. J Clin Psychiatry. 2005; 66: 31-41.

3. Kan CC, Breteler MH, Zitman FG. High prevalence of benzodiazepine dependence in out-patient users, based on the DSM-III-R and ICD-10 criteria. Acta Psychiatr Scand. 1997; 96: 85-93.

4. Lalive AL, Rudolph $\mathrm{U}$, Luscher $\mathrm{C}$, Tan $\mathrm{KR}$. Is there a way to curb benzodiazepine addiction? Swiss Med Wkly. 2011; 141: 13277.

5. Prater CD, Zylstra RG, Miller KE. Successful Pain Management for the Recovering Addicted Patient. Prim Care Companion J Clin Psychiatry. 2002; 4: $125-131$.

6. Hawkins EJ, Malte CA, Hagedorn HJ, Berger D, Frank A, Lott A, et al. Survey of Primary Care and Mental Health Prescribers' Perspectives on Reducing Opioid and Benzodiazepine Co-Prescribing Among Veterans. Pain Med. 2017; 18: 454-467.

7. Dooley TP. Treating anxiety with either beta blockers or antiemetic antimuscarinic drugs: A review. Mental Health in Family Medicine. 2015; 11: 89-99.

8. Neftel KA, Adler RH, Kappeli L, Rossi M, Dolder M, Kaser HE, et al. Stage fright in musicians: a model illustrating the effect of beta blockers. Psychosom Med. 1982; 44: 461-469.

9. Kraus ML, Gottlieb LD, Horwitz RI, Anscher M. Randomized clinical trial of atenolol in patients with alcohol withdrawal. N Engl J Med. 1985; 313: 905909.

10. Horwitz RI, Gottlieb LD, Kraus ML. The efficacy of atenolol in the outpatient management of the alcohol withdrawal syndrome. Results of a randomized clinical trial. Arch Intern Med. 1989; 149: 1089-1093.

11. Gottlieb LD, Horwitz RI, Kraus ML, Segal SR, Viscoli CM. Randomized controlled trial in alcohol relapse prevention: role of atenolol, alcohol craving, and treatment adherence. J Subst Abuse Treat. 1994; 11: 253-258.

12. Ekeberg O, Kjeldsen SE, Greenwood DT, Enger E. Effects of selective beta-adrenoceptor blockade on anxiety associated with flight phobia. J Psychopharmacol. 1990; 4: 35-41.

13. Houde A. Scopolamine: A Physiological and Clinical Study. The American Journal of Clinical Medicine. 1906; 13: 365-367.

14. Witkin JM, Overshiner C, Li X, Catlow JT, Wishart GN, Schober DA, et al. M1 and $\mathrm{m} 2$ muscarinic receptor subtypes regulate antidepressant-like effects of the rapidly acting antidepressant scopolamine. J Pharmacol Exp Ther. 2014; 351: 448-456.
Ann Depress Anxiety - Volume 4 Issue 2 - 2017 ISSN : 2381-8883 | www.austinpublishing group.com Dooley et al. () All rights are reserved Acting Beta Blocker - Scopolamine Combination Drug. Ann Depress Anxiety. 2017; 4(2): 1088. 